\title{
Should Clinicians Split or Lump Psychiatric Symptoms? The Structure of Psychopathology in Two Large Pediatric Clinical Samples from England and Norway
}

\author{
Lorena Fernández de la Cruz 1,2 (C) Pablo Vidal-Ribas ${ }^{2,3} \cdot$ Nada Zahreddine $^{2,4} \cdot$ Børge Mathiassen $^{5}$. \\ Per Håkan Brøndbo ${ }^{5} \cdot$ Emily Simonoff $^{2} \cdot$ Robert Goodman $^{2} \cdot$ Argyris Stringaris $^{3}$
}

Published online: 14 December 2017

(c) The Author(s) 2017. This article is an open access publication

\begin{abstract}
It has been suggested that the structure of psychiatric phenomena can be reduced to a few symptom dimensions. These proposals, mainly based on epidemiological samples, may not apply to clinical populations. We tested the structure of psychiatric symptoms across two pediatric clinical samples from England $(\mathrm{N}=8434)$ and Norway $(\mathrm{N}=5866)$. Confirmatory factor analyses of the parent-reported Strengths and Difficulties Questionnaire (SDQ) evaluated the relative fit of several models, including a first-order model, a second-order model with the widely-established broad symptom dimensions of internalizing-externalizing, and two bi-factor models capturing a general psychopathology factor. Predictive value of the SDQ subscales for psychiatric disorders was examined. A first-order five-factor solution better fit the data. The expected SDQ subscale(s) related best to the corresponding psychiatric diagnosis. In pediatric clinical samples, a granular approach to psychiatric symptoms where several dimensions are considered seems to fit the data better than models based on lumping symptoms into internalizing/externalizing dimensions.
\end{abstract}

Keywords Nosology $\cdot$ Factor structure $\cdot$ Construct validity $\cdot$ Predictive value $\cdot$ Strengths and Difficulties Questionnaire

Lorena Fernández de la Cruz and Pablo Vidal-Ribas are joint first authors.

Electronic supplementary material The online version of this article (https://doi.org/10.1007/s10578-017-0777-1) contains supplementary material, which is available to authorized users.

Lorena Fernández de la Cruz

lorena.fernandez.de.la.cruz@ki.se

1 Department of Clinical Neuroscience, Child and Adolescent Psychiatry Research Center, Karolinska Institutet, Gävlegatan 22 (Entré B), Floor 8, 11330 Stockholm, Sweden

2 Department of Child and Adolescent Psychiatry, Institute of Psychiatry, Psychology \& Neuroscience, King's College London, London, UK

3 Mood Brain \& Development Unit, Emotion and Development Branch, National Institute of Mental Health, Bethesda, MD, USA

4 Department of Psychiatry, Saint-Joseph University, Beirut, Lebanon

5 Regional Centre for Child and Youth Mental Health and Child Welfare - North, UiT The Arctic University of Norway, Tromsō, Norway

\section{Introduction}

It has been suggested that the structure of psychiatric phenomena can be reduced to a few dimensions of symptoms. For example, Kendler et al. [1] assessed a cohort of more than 5600 adult twins from a population-based registry using DSM diagnostic criteria and concluded that genetic risk factors predispose to two broad groups of internalizing and externalizing disorders. In another instance, Wright et al. [2] compared the fit of categorical, continuous, and hybrid (i.e., combined categorical and continuous) models of syndromes in an adult epidemiological sample $(\mathrm{N}=8841)$, assessed via structured clinical interviews, and found that the best fitting higher-order model of these syndromes grouped them into three broad spectra: internalizing, externalizing, and psychotic experiences. In turn, Lahey et al. [3] examined the structure of psychopathology-also assessed using structured interviews - in an epidemiological sample of individuals aged 18-65 and found that a bi-factor model in which every mental disorder loaded in a general factor, in addition to the externalizing, distress, and fears factors, presented the best fit for the data. That is, whereas each of the three group 
factors (externalizing, distress, and fears) accounted well for the correlations among the specific mental disorders that loaded most strongly on those factors, a general dimension captured what all the examined disorders shared in common. Similarly, Caspi and colleagues [4] explored the structure of psychopathology in a longitudinal study that repeatedly assessed individuals from a birth cohort at 18, 21, 26, 32, and 38 years of age, and concluded that psychiatric disorders (assessed via structured interviews) could be explained by three higher-order factors (internalizing, externalizing, and thought disorders), but also found evidence supporting a bi-factor model with one general overriding dimensiontermed the " $p$ factor" - that captured individuals' propensity to develop any and all forms of common psychopathologies over and above individual dimensions for each psychiatric disorder, and the three higher-order factors.

Studies in epidemiological samples of children and adolescents have yielded similar results. For example, in a large population-based sample of young people $(\mathrm{N}=18,222)$, Goodman, Lamping, and Ploubidis [5] used confirmatory factor analysis (CFA) to compare the relative fit of three alternative factor structures for the Strengths and Difficulties Questionnaire (SDQ) [6, 7]. The authors concluded that a second-order model with internalizing and externalizing factors (along with a prosocial subscale factor) fitted the data better than a first-order model with the five hypothesized SDQ subscales. These two models had a substantially better fit than the third alternative: a first-order model with internalizing, externalizing, and prosocial factors. These results replicated the classical internalizing-externalizing approach to the structure of child psychopathology [8]. However, Goodman et al. [5] discussed that, while this factorial solution is probably justified in low-risk samples, a five-factor model might be more appropriate in high-risk populations and clinical samples. Yet, this remains to be tested.

It is argued that "lumping" of psychiatric symptoms into broader dimensions can generate models with several advantages. First, they seem to conform to the genetic architecture of psychiatric symptoms given that individual and aggregate molecular genetic risk factors have been found to be shared among a range of psychiatric disorders that are treated as distinct categories in clinical practice [9]. Second, such a parsimonious model of psychopathology could account for the high rates of comorbidity observed among individuals with mental disorders [10]. However, the above-mentioned findings are based on epidemiological samples which, while offering the benefit of being unbiased by referral practices and being generally larger than clinical samples, may not reflect what is seen in clinical practice.

Indeed, in the rare instance where psychiatric symptoms have been analyzed in clinical samples, it has not been possible to group the data in such a reduced number of dimensions. For example, in adults, Kotov et al. [11] found that the best-fitting model for their sample of 2900 outpatients seeking psychiatric treatment was a five-factor solution, including internalizing, externalizing, thought disorder, somatoform, and antagonism dimensions, which fit the data better than a seven-factor model based on the DSM-IV, an internalizing-externalizing model, a three-factor model with an additional somatoform dimension, and an alternative four-factor model which included the previous three dimensions and additionally placed the psychosis, manic episode, and cluster A traits from the internalizing group into a thought disorder dimension. In a sample of German children and adolescents, Becker et al. [12, 13] subjected the items of the SDQ to CFA, and demonstrated a good fit of the original five-factor model both for the parent $(\mathrm{N}=543)$ and the self-reported $(\mathrm{N}=214)$ measure. Interestingly, they also employed exploratory factor analysis (EFA) and the results highly converged with the five original SDQ subscales. The differing evidence between epidemiological and the few studied clinical samples is a clear example of how factorial structure is determined by the type of sample (i.e., the number of ill individuals that these contain and the types of problems that they have) [14], and in this specific case, it suggests that less parsimonious dimensional structures may better reflect the reality of individuals with psychiatric disorders.

In fact, models based on "splitting"-rather than lumping-also have considerable support, particularly in relation to clinical variables. For example, whilst genetic etiology may be largely shared between various anxiety disorders, their distinction may be important in relation to family history, neurobiology, and treatment response [15]. Perhaps even more strikingly, whilst many of the most parsimonious models of psychopathology would consider hyperactivity and conduct problems or irritability as a joint entity, their distinction has key implications for treatment and course. Attention-deficit/hyperactivity disorder (ADHD) symptoms do not respond to parenting interventions [16], whilst conduct and oppositional problems do [16, 17]; conversely, stimulants show large effect sizes particularly for hyperactivity, impulsivity, and inattention, yet less so for irritability and related behaviors $[18,19]$. It is therefore crucial to examine whether the structure of psychopathology found in epidemiological samples applies to clinical samples.

Additionally, it remains a matter of debate whether distinct structures of symptoms can have an impact in the prediction of psychiatric outcomes, and whether this is influenced by the type of sample. Results from epidemiological samples have yielded a moderate to high level of agreement between SDQ-generated diagnoses and corresponding clinical diagnoses $[20,21]$. More recently, Goodman et al. [5] found that a second-order structure of the SDQ with internalizing, externalizing, and prosocial factors showed clear convergent and discriminant validity when predicting 
clinical disorders even at the lowest SDQ scores. By contrast, the five SDQ subscales only showed convergent and discriminant validity in children with high scores on those subscales, especially for behavioral and hyperactivity problems. These findings would also support the hypotheses that less parsimonious models account for symptoms in clinical samples.

Results of predictive validity in clinical samples are mixed. Becker and colleagues $[12,13]$ showed that the total difficulties score of the SDQ was a good predictor of any axis I diagnosis; furthermore, they found that the subscales of the SDQ predicted well their matching diagnostic categories in their clinical sample. However, in a more recent study by Brøndbo et al. [22] on a Norwegian clinical sample, the SDQ was considered insufficient for clinical purposes. The authors also concluded that the SDQ was better in detecting the presence of "any diagnosis" rather than more specific ones and, conversely, was better at ruling out specific diagnoses rather than "any diagnosis." Given these results, larger clinical samples might be needed to ascertain the predictive value of distinct symptom structures.

While previous studies have examined a first-order fivefactor model in young clinical samples [12, 13], no studies to date have offered a comparison of alternative models described in the literature. In addition, no studies in clinical samples have provided with a cross-country validation of these models, hence limiting the generalizability of previous results. Moreover, while several studies have examined the prediction of psychiatric disorders using different symptoms dimensions, no studies have tested whether these predictions hold when these disorders co-occur. The current study tries to fill these gaps in the literature with the following three aims.

First, using CFA in two independent clinical samples from England and Norway, we examine the relative fit of key alternative models using the SDQ, which is one of the most widely-used instruments to measure child and adolescent psychopathology worldwide [e.g., 23, 24]. For comparability, we test the models that have been comprehensively tested before in large epidemiological samples [3-5]. These include a first-order five-factor model, a second-order model with the widely-established broad symptom dimensions of internalizing-externalizing, and two bi-factor models capturing a general psychopathology factor.

Second, as we employ two large samples from different countries, we examine the measurement invariance of the best fitting model across countries to see whether the same structure is generalizable. This is particularly relevant since differences have been found in the presentation of psychopathological symptoms between these countries [25].

Third, we test the external validity of the dimensions. In particular, we test whether each dimension of symptomseither first- or second-order dimensions-specifically links with psychiatric disorders. Finally, given that comorbidity is typical, we test whether psychopathological symptoms are differently distributed in participants with distinct comorbidities across both samples.

\section{Methods}

\section{Study Setting and Samples}

A clinical sample from London, England (hereafter the England sample) was obtained from the South London and Maudsley (SLaM) NHS Foundation Trust. SLaM is Europe's largest specialist mental health care area. It serves a population of approximately 1.2 million residents of four South London local authorities. The SLaM Biomedical Research Centre (SLaM BRC) Case Register is a data resource containing de-identified electronic records of all secondary and tertiary mental healthcare service users from SLaM. The Clinical Record Interactive Search (CRIS) is a program which automatically and effectively de-identifies full clinical records derived from the electronic clinical records system in SLaM [26], enabling researchers to search and retrieve deidentified data from these electronic medical records which include over 180,000 cases. Of these, approximately 35,000 are receiving active care from SLaM at a given time. The protocol for this case register has been described in detail elsewhere [27]. CRIS has been used in numerous research studies [e.g., 28-30]. For this study, the variable that defined the main cohort was the existence of an SDQ. Other relevant variables were retrieved for the cohort of participants, including month and year of birth (full dates of birth and postcodes were not available to avoid identification of the individuals), gender, and scores on the Development and Well-Being Assessment (DAWBA), if available. These variables are routinely recorded on SLaM electronic patient records in designated fields. CRIS was approved as a dataset for secondary analysis by Oxfordshire Research Ethics Committee C (reference 08/H0606/71). Approval for the present study was sought and obtained from the CRIS Oversight Committee.

The second large clinical sample, from the University Hospital of North Norway (UNN), Norway (hereafter the Norway sample), includes patients from the Child and Adolescent Mental Health Services (CAMHS) at UNN. UNN is a specialist health area serving a population of 190,726 residents of the county municipalities of Troms and the northern part of Nordland. The health trust is covering an area of approximately $31,300 \mathrm{~km}^{2}$. Annually, UNN provides mental health services to about $5 \%$ $(2100 / 42,000)$ of the population aged $0-18$ years. About $60 \%$ of the treated patients are new referrals from general practitioners and the child protection services. The 
CAMHS at UNN consist of six outpatient and one inpatient clinics. All of them include the online version of the DAWBA in the routine clinical assessment. The questionnaire SDQ is an integrated part of DAWBA. All DAWBA data at UNN are stored in a de-identified local CAMHS quality register. The data protection officer at UNN has approved the use of data from the quality register for research purposes.

Main characteristics of both the England and the Norway samples, including sample sizes, main demographics, and clinical characteristics, can be found in Table 1 . In both sites, the cohorts were defined by the presence of an SDQ, independently of the respondent (parent, teacher, or the young patients themselves). However, only the parent-reported SDQ was used in the analyses. The completion rate of the parent-reported SDQ was $81 \%(n=6846)$ in the England sample and $84 \%(n=4940)$ in the Norway sample. Compared to participants without parent-reported SDQ, those participants with completed parent SDQs were older and more likely to be males in both the England (age: $\mathrm{t}(5854)=-18.10, \mathrm{p}<0.0001$; gender: $\left.\chi^{2}=70.85, \mathrm{p}<0.001\right)$ and the Norway sample (age: $\mathrm{t}(8409)=-27.63, \mathrm{p}<0.0001$; gender: $\left.\chi^{2}=86.29, \mathrm{p}<0.001\right)$.
Participants with missing information on DAWBA diagnoses ranged from $25-27 \%$ in the Norway sample and from 97-98\% in the England sample (missing values vary across diagnoses). Those with missing information in diagnoses were more likely to be males and older in the Norway sample; whereas these were younger in the England sample.

\section{Measures}

The SDQ is a 25 -item questionnaire with five hypothesized subscales: emotional problems, peer problems, behavioral problems, hyperactivity, and prosocial behavior, as well as an additional impact scale $[6,7,31]$. Each subscale comprises five questions with three-point response scales ('Not true' $=0$, 'Somewhat true' $=1$, 'Certainly true' $=2$ ), with a subscale score range of $0-10$. Ten of the 25 items are positively worded 'strengths'; these are reverse-scored if they contribute to the emotional, peer, behavioral, or hyperactivity subscales. The SDQ has been validated in Norwegian samples [22, 32]. See http://www.sdqinfo.org for a full description of measure and items.

The SDQ parent-report was used due to a number of reasons. First, parents are the main informants in psychiatric

Table 1 Demographic and clinical characteristics of the South London and Maudsley NHS Foundation Trust and the University Hospital of North Norway samples

\begin{tabular}{|c|c|c|c|c|c|c|c|}
\hline & \multicolumn{2}{|c|}{ England $(\mathrm{N}=8434)$} & \multicolumn{2}{|c|}{ Norway $(\mathrm{N}=5866)$} & \multicolumn{3}{|c|}{ Statistics } \\
\hline & Mean & $\mathrm{Sd}$ & Mean & $\mathrm{Sd}$ & $\mathrm{t}$ & $\mathrm{df}$ & $p$ \\
\hline Age $(\text { years })^{\mathrm{a}}$ & 11.2 & 3.8 & 12.1 & 3.6 & 14.5 & 14,265 & $<.0001$ \\
\hline \multicolumn{8}{|l|}{ SDQ scores ${ }^{\mathrm{b}}$} \\
\hline SDQ total score & 18.3 & 7.7 & 16.3 & 6.6 & 14.6 & 11,871 & $<.0001$ \\
\hline SDQ emotional & 4.7 & 2.8 & 4.4 & 2.7 & 6.8 & 11,868 & $<.0001$ \\
\hline SDQ behavioral & 3.9 & 2.6 & 3.1 & 2.1 & 17.8 & 11,868 & $<.0001$ \\
\hline SDQ hyperactivity & 6.2 & 2.9 & 5.5 & 2.8 & 12.8 & 11,837 & $<.0001$ \\
\hline SDQ peer problem & 3.6 & 2.4 & 3.3 & 2.4 & 5.1 & 11,854 & $<.0001$ \\
\hline SDQ prosocial & 6.5 & 2.6 & 6.9 & 2.2 & 7.5 & 11,868 & $<.0001$ \\
\hline \multirow[t]{2}{*}{ SDQ impact } & 4.4 & 2.9 & 4.4 & 2.6 & 1.4 & 10,418 & .15 \\
\hline & $\mathrm{n}$ & $\%$ & $\mathrm{n}$ & $\%$ & $\chi^{2}$ & $\mathrm{df}$ & $p$ \\
\hline Gender (females) & 3673 & 44 & 2802 & 48 & 24.1 & 1 & $<.001$ \\
\hline \multicolumn{8}{|l|}{ DAWBA diagnoses $^{\mathrm{c}}$} \\
\hline Emotional disorder ${ }^{\mathrm{d}}$ & 186 & 77 & 2116 & 48 & 77.6 & 1 & $<.001$ \\
\hline Behavioral disorder & 150 & 62 & 2209 & 51 & 11.5 & 1 & $<.005$ \\
\hline ADHD & 101 & 46 & 1627 & 37 & 6.9 & 1 & $<.01$ \\
\hline ASD & 31 & 16 & 189 & 4 & 54.8 & 1 & $<.001$ \\
\hline
\end{tabular}

${ }^{a}$ Age range for the England sample 2-18 years; age range for the Norway sample 2-19 years

${ }^{b}$ Number of observations in SDQ scores varies depending on the SDQ subscale (England: 6789-6933; Norway: 4915-4961). Range in SDQ Total Difficulties score is 0-40; range in SDQ subscales is $0-10.1$

${ }^{c}$ All disorders are defined with DAWBA bands (>2). Information about diagnoses was available for $3 \%$ of the England sample and $80 \%$ of the Norway sample

${ }^{\mathrm{d}}$ Any depressive or anxiety disorder

${ }^{\mathrm{e}}$ Conduct disorder or oppositional defiant disorder 
services for young people. Second, parent reports have shown to be a better predictor of psychiatric outcomes than self-reports [31,33]. Third, by using parent report, we could enrich the samples by including children aged 10 years or younger in the analyses, as self-report versions of the SDQ are not available for these ages.

The DAWBA [34] is a detailed psychiatric interview administered by lay interviewers to parents and youth, with a briefer questionnaire for teachers. Each section begins with structured questions that cover the operationalized diagnostic criteria for DSM-IV [35]. Structured questions are supplemented by open-ended questions which record verbatim a respondent's own description of problem areas. Clinicians review the close and open responses from all informants, identifying discrepancies within or between informants, and using the content, length, and tone of the transcripts to interpret conflicting information [36]. On this basis, raters decide whether a particular child meets all the relevant DSM-IV criteria for an operationalized mental disorder. Raters can also assign 'Not Otherwise Specified' disorder, for example 'behavioral disorder, not otherwise specified' when children have substantial impairment from symptoms which do not quite meet operationalized criteria. In this paper, we group the mental disorders into emotional disorders (including anxiety and depressive disorders); behavioral disorders (including oppositional defiant and conduct disorders); ADHD; and autism spectrum disorders (ASD; including autism and Asperger's syndrome). Participants were assigned a positive diagnosis if they scored 3 or higher in the relevant DAWBA bands [37], as previously described [38]. In British samples, the DAWBA has shown to have good inter-rater reliability (e.g., kappa $=0.86$ for inter-rater agreement for 'any mental disorder' in an epidemiological sample) [39]. It also has good validity as judged against case-notes diagnoses, performs well in differentiating clinic/community samples, and shows strong associations with risk factors, service use, and 3-year prognosis [20,39]. See http://www.dawba.com for additional information about the DAWBA.

\section{Statistical Analyses}

\section{Confirmatory Factor Analyses}

We used CFA to evaluate and compare the relative fit of four alternative factor structures for the parent SDQs in the England and Norway samples. These were: (1) a first-order model with the correlated five hypothesized SDQ factors (Model 1) [6]; (2) a second-order model with the SDQ emotional problems and peer problems subscales loading on an 'internalizing' factor, plus the SDQ hyperactivity and behavioral problems subscales loading on an 'externalizing' factor, with both second-order factors correlating with a firstorder factor composed by the items of the SDQ prosocial behavior subscale (Model 2); (3) a bi-factor model including a single general factor and the five SDQ subscales as group factors (Model 3); and (4) a bi-factor model including a single general factor and an externalizing, an internalizing, and a prosocial behavior factor as group factors (Model 4; see Fig. 1). Models 1 and 2 have shown to best fit the data in epidemiological samples using the same instrument [5], whereas Models 3 and 4 examine the existence of a general psychopathological factor similar, though not identical, to that found previously [3, 4].We performed the CFA in MPlus version 5.1, using a multivariate probit analysis for ordinal data $[40,41]$ and estimating model fit using the Weighted Least Squares, Mean, and Variance adjusted (WLSMV) estimator. We followed common practice in reporting multiple indices of fit, namely the Comparative Fit Index (CFI), the Tucker Lewis Index (TLI), and the Root Mean Square Error of Approximation (RMSEA) [42, 43]. To consider a model as showing 'acceptable' fit, we required a CFI $>0.90$, TLI $>0.90$, and RMSEA $<0.08$; to consider a model as showing 'good' fit, we required a CFI $>0.95$, TLI $>0.95$, and RMSEA $<0.06$ [43]. $\chi^{2}$ difference test was used to compare nested models, where improvements in model fit by the nested-less constrained and more parsimonious-model are tested. We also compared models using the Akaike Information Criteria (AIC) and the Bayesian Information Criteria (BIC), where lower values indicate better fit [44, 45]. Unlike $\chi^{2}$, AIC and BIC penalize per increasing number of estimated parameters, thus avoiding overfitting models. Where models showed acceptable fit on some indices but not on others, we allowed correlations between the unique variances of some individual items within the same factor, selecting these item pairs using MPlus' modification indices. Such minor model modifications can improve model fit by increasing the proportion of variance explained, but do not change the substantive conclusions regarding the adequacy of a hypothesized factor structure in describing a set of data [46].

\section{Measurement Invariance}

Measurement invariance (MI) is present when a specific instrument (e.g., SDQ) measures the same construct across different groups [47]. If MI is established, then it can be confirmed that the participants across all groups interpret the individual items, as well as the underlying latent factors, in the same way. This is particularly important in crosscultural research when comparing results across different cultures and people speaking different languages (in this case, English and Norwegian). Conversely, failure to prove MI indicates that groups interpret the items differently and, as a consequence, factor means cannot be compared in a meaningful way $[48,49]$. 

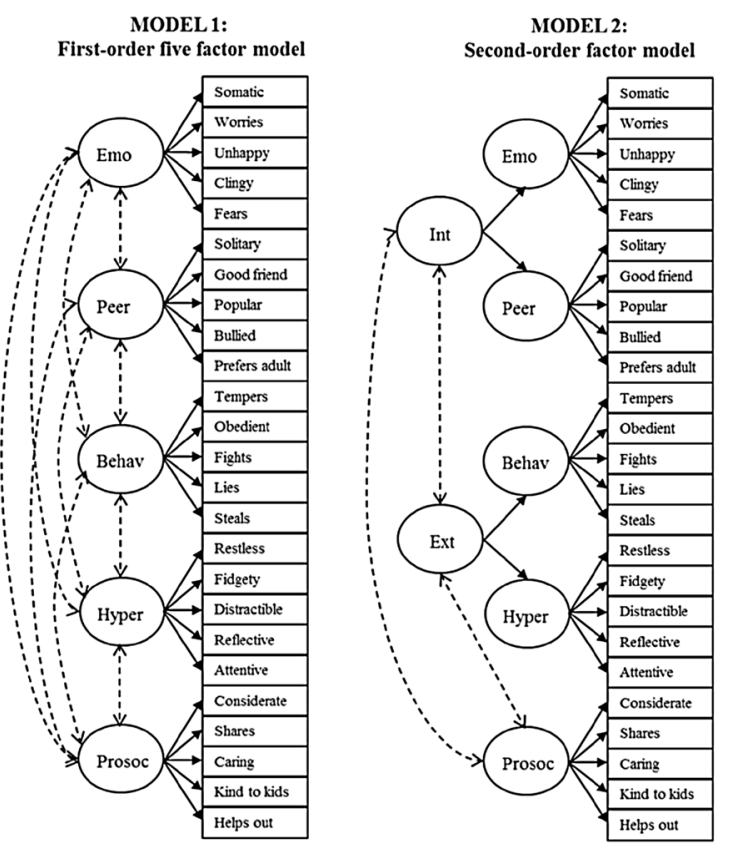

Fig. 1 Models to be tested in the confirmatory factor analyses. Solid lines represent factor loadings. Dashed lines represent correlations. Behav behavioral problems, Emo emotional problems, Ext external-

Here we tested MI across gender (males vs. females), groups of age ( 2 to 11 year-old children vs. 12 to 18 yearold adolescents), and countries (England vs. Norway) to examine whether the results from the CFA were consistent across these groups and allow comparisons between the two different samples included in the study. MI was tested in MPlus version 5.1 using WLSMV estimator, and theta parameterization.

MI examines the change in the goodness-of-fit indices (GFI) when cross-group constraints are imposed on a measurement model in a hierarchical set of steps [50, 51]. Configural, metric, scalar, and measurement error invariance were tested. Configural invariance refers to whether the same CFA is valid in each group. Metric invariance-also called weak factorial invariance-concerns the equivalence of the factorial loadings across groups. Scalar (or strong factorial) invariance is assumed when the item intercepts and the factor loadings are equally constrained across groups. Finally, when testing measurement error (or strict factorial) invariance, also the variances of the residuals are constrained across groups.

The Chi square difference test is widely used to compare nested models [52]. However, this test yields significant differences in large sample sizes [53]. Therefore, we compared models on the basis of changes in CFI $(\Delta \mathrm{CFI})$ as suggested by Cheung and Rensvold [47]. A value of $\Delta$ CFI higher than or equal to -0.01 indicates that the null hypothesis of invariance should not be rejected.

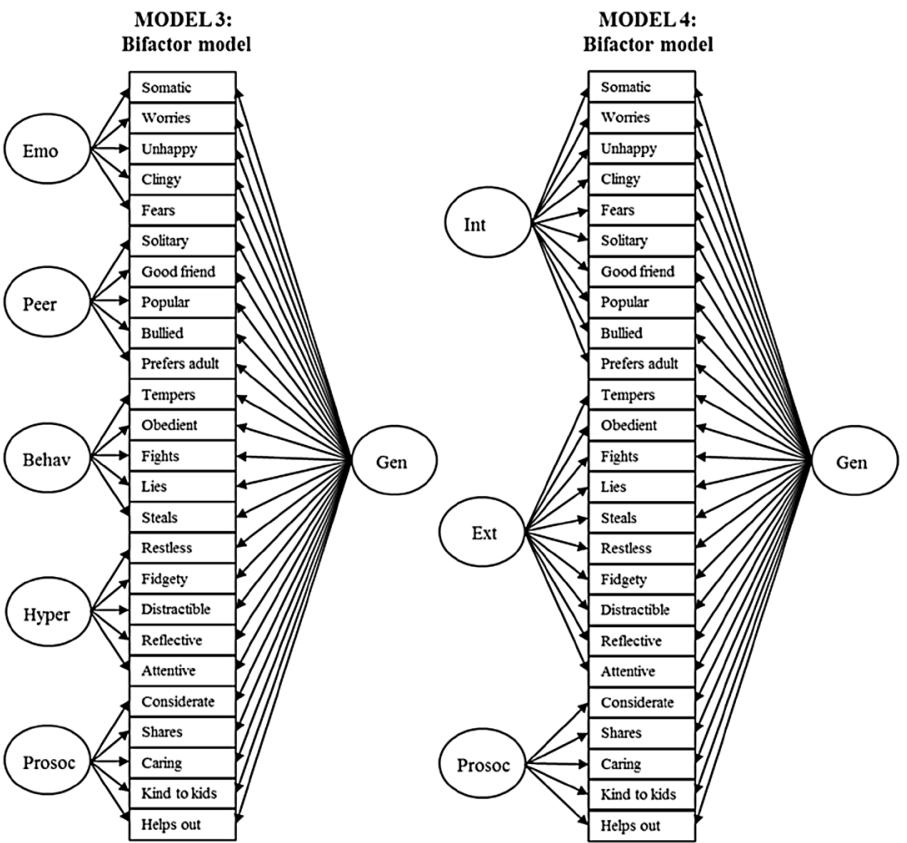

izing, Gen general factor, Hyper hyperactivity problems, Int internalizing, Peer peer problems, Prosoc prosocial problems

\section{Predictive Value of the SDQ Subscales}

We performed a series of logistic regression analyses in Stata 11 using DAWBA diagnoses for any emotional disorder, any behavioral disorder, ADHD, or ASD as outcomes. All five hypothesized SDQ subscales were entered in the model as predictors of interest. In a second step, we employed internalizing and externalizing problems as predictors as a comparison. Given the high rates of missing values in DAWBA diagnoses in the England sample, we replicated these analyses in MPlus using Full Information Maximum Likelihood (FIML). Maximum likelihood uses all the available data to generate parameter estimates without the need to discard cases or fill in the data prior to analysis. This method is currently regarded as the "state of the art" missing data technique [54] given that requires of less strict assumptions about the mechanism that led to missing data and generally produces more accurate estimates than traditional missing data handling techniques (e.g., discarding cases).

In addition, given that comorbidity was frequent across both samples $(56 \%$ of participants with a diagnosis met criteria for at least a second diagnosis), we tested whether SDQ subscales were differently distributed among groups of comorbidity. We focused these analyses in participants with two diagnoses, who represented $63 \%$ of those with comorbidities. We performed comparisons across three different groups: emotional + conduct disorder (CD) 
$(\mathrm{n}=443)$, emotional + ADHD $(\mathrm{n}=123)$, and CD + ADHD $(n=524)$. Since rates of ASD were low compared to other disorders $(\mathrm{n}=14 \mathrm{CD}+\mathrm{ASD} ; \mathrm{n}=12$ emotional $+\mathrm{ASD}$; and $n=5$ ADHD + ASD), comorbid ASD groups were excluded from the analyses. Levene's robust test statistic (W0) for the equality of variances showed that variance of SDQ emotional, peer problems and prosocial subscale scores did not differ across comorbidity groups (all $p>0.300$ ), and these were examined with analysis of variance (ANOVA) with Bonferroni correction. Variances of SDQ behavioral (W0 $=7.98, \mathrm{p}<0.001$ ) and hyperactivity (W0 $=17.33, \mathrm{p}<0.001$ ) subscales scores differed across groups, and these were examined with Welch ANOVA with Bonferroni correction.

In all analyses, we reverse-scored the SDQ prosocial subscale in order to facilitate comparisons of effect sizes across subscales.

\section{Results}

\section{Confirmatory Factor Analyses}

The first-order five-factor model (Model 1) converged well. However, the second-order factor model (Model 2) yielded Heywood cases in both samples (i.e., negative residual variances and correlations greater than 1.00). Specifically, in the England sample, the first-order factor 'peer' showed a negative residual variance and a standardized association greater than 1.00 with the second-order factor 'internalizing'. In contrast, in the Norway sample, the factor 'behavioral' showed the same pattern with the 'externalizing' factor. These findings indicate an overlapping of variance between the first- and second-order factors such that discriminant validity between these two constructs is indistinguishable. In other words, the secondorder factor predicts almost perfectly one of the first-order
Fig. 2 Second-order factor models used in the confirmatory factor analyses. Solid lines represent factor loadings. Dashed lines represent correlations. Behav behavioral problems, Emo emotional problems, Ext externalizing, Hyper hyperactivity problems, Int internalizing, Peer peer problems, Prosoc prosocial problems

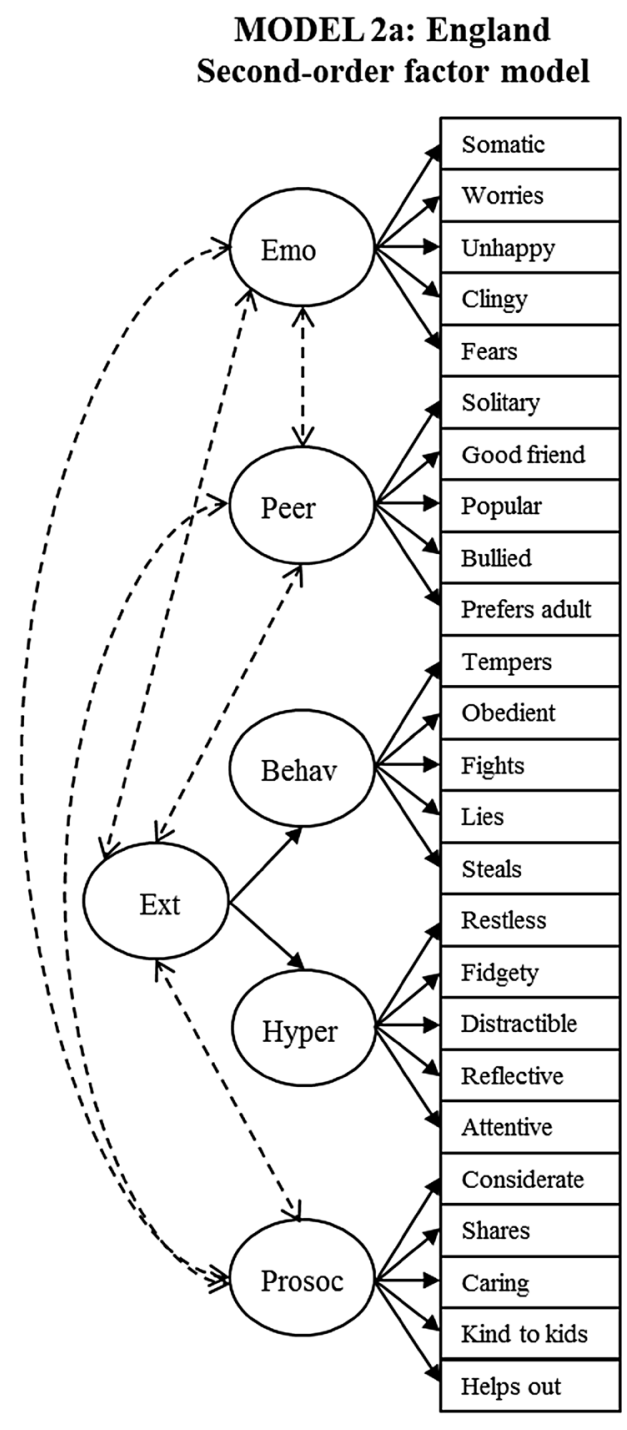

MODEL 2a: England Second-order factor model
MODEL 2b: Norway Second-order factor model

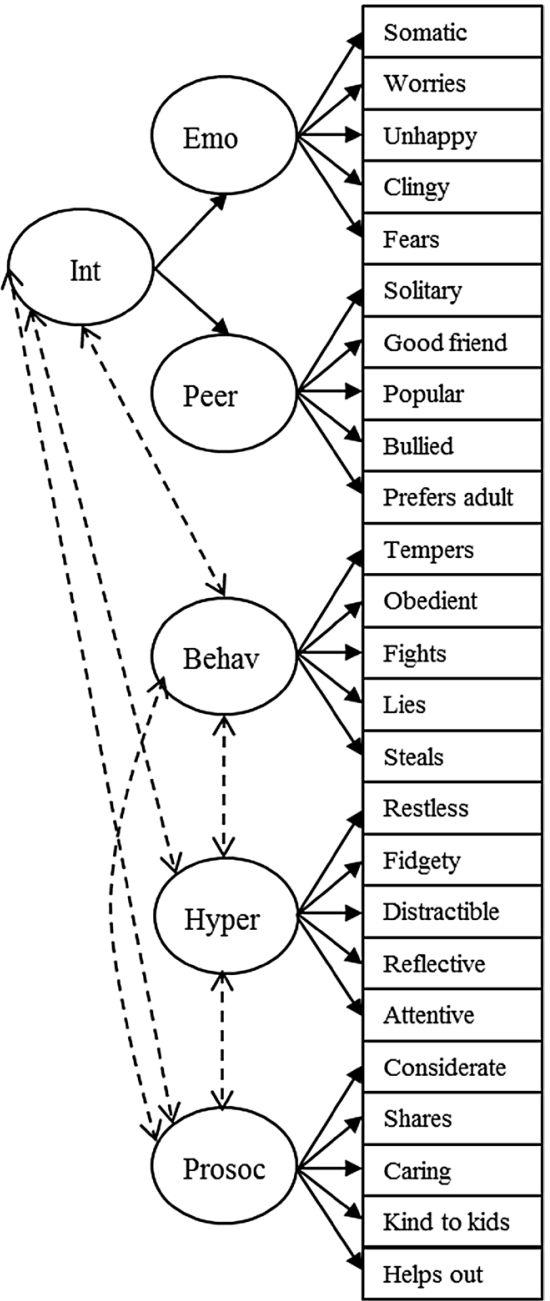


factors [55]. Given these results, we modified the models as shown in Fig. 2 (Model 2 modified to Models $2 \mathrm{a}$ and $2 \mathrm{~b}$ for the England and Norway sample, respectively).

Model 3 (five group factors and a general bi-factor) only converged in the England sample and showed that the variance of the general factor was very low (0.011). In addition, the standardized loadings of the emotional items into the general factor were also low, ranging from 0.07 to 0.27 . In order to make Model 3 converge in the Norway sample, as well as Model 4 in both samples, we had to fix variances of all factors at 1 and free the estimation of the first indicator. In all cases, the standardized loadings of the emotional items into the general factor were low, ranging from 0.00 to 0.31 .

As seen in Table 2, the five hypothesized SDQ factors (Model 1) and the second-order factor model with additional 'internalizing' or 'externalizing' factors (Models 2a and $2 \mathrm{~b}$ ), showed acceptable fit in all indices (CFI $>0.85$, TLI $>0.90$, RMSEA < 0.08). In contrast, both bi-factor models only showed acceptable fit in CFI and RMSEA in the England sample.

Comparison of nested Models 1 and 2 yielded significant $\chi^{2}$ difference tests (England: $\Delta \chi 2=17.74 ; \Delta \mathrm{df}=2$; $\mathrm{p}=0.0001$. Norway: $\Delta \chi 2=73.39 ; \Delta \mathrm{df}=2 ; \mathrm{p}<0.0001)$, favoring Model 1 (five first-order factors) compared to Models $2 \mathrm{a}$ and $2 \mathrm{~b}$ across both samples. However, in the comparison of bi-factor models (Models 3 and 4) with Models 1 and 2, computation of $\Delta \chi^{2}$ was not possible due to the singular matrix during the computation process.

Overall, and taking all the goodness-of-fit indices into account, Model 1 (first-order five-factor solution) was the one that showed the best fit to the data among all the models tested.

\section{Measurement Invariance}

In order to test whether our results were comparable across gender, age, and site, we tested the MI. Overall, and in line with our hypothesis, MI held for all comparisons by gender, age, and country for the model that had shown the best fit in the previous analyses (Model 1).

In all cases, $\Delta$ CFI was equal to or higher than -0.01 (range $=-0.010$ to 0.017 ) when compared to the preceding model, which suggests that the model should not be rejected (Table 3).

\section{Predictive Value of the SDQ Subscales}

Finally, we wanted to test whether each dimension of symptoms of the SDQ specifically linked with diagnostic outcomes. The results in the invariance testing allowed us to merge both clinical samples in order to predict clinical diagnoses.

Table 4 shows which subscales (including internalizing and externalizing scores) had the largest effect on the odds of receiving a DAWBA diagnosis. In all cases, the expected subscale(s) always had the largest point estimates of effect size. These point estimates were also substantially and significantly larger than the next-largest estimates, except for the peer and prosocial subscales predicting ASD, which were not significantly different from the hyperactivity or externalizing subscales. The prediction of emotional and behavioral disorders was stronger using the five-subscale model as evidenced by the non-overlap of the $95 \%$ confidence interval estimates with the three-subscale model (emotional: $\mathrm{t}=3.22, \mathrm{p}=0.0013$; behavioral: $\mathrm{t}=4.03, \mathrm{p}=0.0001$ ). The analyses using FIML, which included all the available
Table 2 Model fit in confirmatory factor analyses of the England and Norway samples' strengths and difficulties questionnaires (SDQs)

\begin{tabular}{|c|c|c|c|c|c|c|c|}
\hline Sample & Model $^{\mathrm{a}}$ & $\chi^{2}$ & $\mathrm{df}$ & $p$ & CFI & TLI & RMSEA \\
\hline \multirow[t]{4}{*}{ England $^{\mathrm{b}} \mathrm{N}=6912$} & Model 1 & 7517.16 & 256 & $<0.0001$ & 0.923 & 0.909 & 0.064 \\
\hline & Model 2a & 7367.15 & 258 & $<0.0001$ & 0.924 & 0.912 & 0.063 \\
\hline & Model 3 & 9082.67 & 250 & $<0.0001$ & 0.906 & 0.887 & 0.071 \\
\hline & Model $4^{\mathrm{d}}$ & 8460.17 & 250 & $<0.0001$ & 0.912 & 0.895 & 0.069 \\
\hline \multirow[t]{4}{*}{ Norway $^{\mathrm{c}} \mathrm{N}=4961$} & Model 1 & 6104.11 & 254 & $<0.0001$ & 0.921 & 0.907 & 0.068 \\
\hline & Model 2b & 6089.42 & 256 & $<0.0001$ & 0.922 & 0.908 & 0.068 \\
\hline & Model $3^{\mathrm{d}}$ & 8446.68 & 250 & $<0.0001$ & 0.890 & 0.868 & 0.081 \\
\hline & Model $4^{\mathrm{d}}$ & 9533.07 & 250 & $<0.0001$ & 0.875 & 0.850 & 0.087 \\
\hline
\end{tabular}

${ }^{a}$ Model 1 is displayed in Fig. 1; Models 2a, and 2b are displayed in Fig. 2

${ }^{\mathrm{b}}$ England sample minor modifications: allowing correlation between the unique variance of (clingy and unhappy) (steals and lies) (fidgety and restless) (reflective and restless) (reflective and fidgety) (persistent and restless) (persistent and fidgety) (persistent and distractible) (volunteers and helpful)

${ }^{c}$ Norway sample minor modifications: allowing correlation between the unique variance of (clingy and fears) (clingy and unhappy) (steals and lies) (distractible and restless) (distractible and fidgety) (restless and fidgety) (reflective and fidgety) (reflective and restless) (persistent and restless) (persistent and fidgety) (persistent and distractible)

${ }^{\mathrm{d}}$ Factor variances had to be fixed at 1 to make the model converge 
Table 3 Fit indices for invariance test of the five-factor model

\begin{tabular}{|c|c|c|c|c|c|c|c|}
\hline Sample/model & $\chi^{2}$ & df & $p$ & CFI & TLI & RMSEA & $\Delta \mathrm{CFI}$ \\
\hline \multicolumn{8}{|l|}{ England } \\
\hline \multicolumn{8}{|c|}{ Gender: males $(n=4066)$ versus females $(n=2844)$} \\
\hline Configural invariance & 5560.73 & 279 & $<0.0001$ & 0.879 & 0.934 & 0.074 & - \\
\hline Metric invariance & 5819.13 & 295 & $<0.0001$ & 0.874 & 0.935 & 0.074 & -0.005 \\
\hline Scalar invariance & 5841.17 & 304 & $<0.0001$ & 0.873 & 0.937 & 0.073 & -0.001 \\
\hline Error variance invariance & 5082.82 & 289 & $<0.0001$ & 0.890 & 0.942 & 0.069 & 0.017 \\
\hline \multicolumn{8}{|c|}{ Age: $2-11$ years old $(n=3864)$ versus $12-18$ years old $(n=3048)$} \\
\hline Configural invariance & 5967.37 & 276 & $<0.0001$ & 0.876 & 0.934 & 0.077 & - \\
\hline Metric invariance & 6160.22 & 290 & $<0.0001$ & 0.872 & 0.935 & 0.077 & -0.004 \\
\hline Scalar invariance & 6531.73 & 299 & $<0.0001$ & 0.864 & 0.933 & 0.078 & -0.008 \\
\hline Error variance invariance & 5818.53 & 285 & $<0.0001$ & 0.879 & 0.938 & 0.075 & 0.015 \\
\hline \multicolumn{8}{|l|}{ Norway } \\
\hline \multicolumn{8}{|c|}{ Gender: males $(n=2708)$ versus females $(n=2253)$} \\
\hline Configural invariance & 4196.60 & 262 & $<0.0001$ & 0.897 & 0.934 & 0.078 & - \\
\hline Metric invariance & 4396.89 & 274 & $<0.0001$ & 0.892 & 0.934 & 0.078 & -0.005 \\
\hline Scalar invariance & 4410.24 & 281 & $<0.0001$ & 0.892 & 0.936 & 0.077 & 0.000 \\
\hline Error variance invariance & 4085.97 & 278 & $<0.0001$ & 0.901 & 0.940 & 0.074 & 0.009 \\
\hline \multicolumn{8}{|c|}{ Age: $2-11$ years old $(n=2245)$ versus $12-18$ years old $(n=2716)$} \\
\hline Configural invariance & 4475.85 & 260 & $<0.0001$ & 0.887 & 0.929 & 0.081 & - \\
\hline Metric invariance & 4759.46 & 272 & $<0.0001$ & 0.880 & 0.928 & 0.082 & -0.007 \\
\hline Scalar invariance & 5018.10 & 280 & $<0.0001$ & 0.873 & 0.926 & 0.083 & -0.007 \\
\hline Error variance invariance & 4859.67 & 277 & $<0.0001$ & 0.878 & 0.928 & 0.082 & 0.005 \\
\hline \multicolumn{8}{|l|}{ England and Norway } \\
\hline \multicolumn{8}{|c|}{ Country: England $(n=6912)$ versus Norway $(n=4961)$} \\
\hline Configural invariance & 9855.78 & 273 & $<0.0001$ & 0.889 & 0.936 & 0.077 & - \\
\hline Metric invariance & 10442.59 & 287 & $<0.0001$ & 0.883 & 0.935 & 0.077 & -0.006 \\
\hline Scalar invariance & 11291.82 & 296 & $<0.0001$ & 0.873 & 0.932 & 0.079 & -0.010 \\
\hline Error variance invariance & 11359.88 & 289 & $<0.0001$ & 0.872 & 0.930 & 0.080 & -0.001 \\
\hline
\end{tabular}

data $(\mathrm{N}=11,918-11,925)$, replicated the previous results; i.e., emotional disorder was best predicted by SDQ emotional score $(\mathrm{OR}=3.65, \mathrm{p}<0.001), \mathrm{CD}$ was best predicted by SDQ behavioral score $(\mathrm{OR}=6,90, \mathrm{p}<0.001)$, ADHD was best predicted by SDQ hyperactivity score $(\mathrm{OR}=6,64$, $\mathrm{p}<0.001$ ), and ASD was best predicted by SDQ peer problems and prosocial scores $(\mathrm{OR}=2.48$ and 2.00, respectively, both $\mathrm{p}<0.001)$.

Analyses of variance with those participants with comorbidities showed a pattern similar to that obtained using the logistic regression analyses. Scores of SDQ emotional subscale were higher in the two groups with emotional disorder compared to the CD + ADHD (all p values $<0.001$ ). SDQ behavioral subscale scores were higher in the two groups with CD compared to Emotional + ADHD (all p values $<0.001)$, as well as higher in CD + ADHD than in $\mathrm{CD}+$ Emotional $(\mathrm{p}<0.001)$. SDQ hyperactivity scores were higher in the two groups with ADHD, compared to the group with $\mathrm{CD}+$ Emotional (all $\mathrm{p}<0.001)$. CD + ADHD also scored higher in hyperactivity than the emotional + ADHD group $(\mathrm{p}<0.001)$. There were no differences in SDQ peer problems between groups. Reversed SDQ prosocial scores were higher in the two groups with CD compared with Emotional + ADHD (all p values $<0.001$ ). Standardized SDQ subscale scores for each comorbidity group are depicted in Fig. 3.

\section{Discussion}

In this study, we used data from 14,209 children and adolescents from clinical settings (8343 from England and 5866 from Norway) to explore the symptom structure of their psychiatric symptoms using a widely used psychometric tool, the SDQ. Results showed that a five-factor structure presented the best fit for the data in both samples and was superior to second order factor models with additional 'internalizing' and 'externalizing' factors, as well as to two bi-factor models that accounted for a general factor. This finding contrasts with those of previous epidemiological studies [e.g., 5], suggesting that psychiatric disorders present with unique phenotypic characteristics 
Table 4 Association of the strengths and difficulties questionnaire (SDQ) subscales with the development and well-being assessment (DAWBA) diagnoses at baseline

\begin{tabular}{|c|c|c|c|c|}
\hline & $\begin{array}{l}\text { Emotional DAWBA } \\
\text { diagnosis }\end{array}$ & $\begin{array}{l}\text { Behavioral DAWBA } \\
\text { diagnosis }\end{array}$ & $\begin{array}{l}\text { ADHD } \\
\text { DAWBA diagnosis }\end{array}$ & $\begin{array}{l}\text { ASD } \\
\text { DAWBA diagnosis }\end{array}$ \\
\hline & Odds ratio $(95 \% \mathrm{CI})$ & Odds ratio $(95 \% \mathrm{CI})$ & Odds ratio $(95 \% \mathrm{CI})$ & Odds ratio $(95 \% \mathrm{CI})$ \\
\hline $\begin{array}{l}\text { England and Norway } \\
\text { samples }\end{array}$ & $n=4601$ & $n=4547$ & $n=4542$ & $n=4447$ \\
\hline Emotional (E) & $3.65(3.36,3.98) * * *$ & $0.97(0.89,1.06)$ & $0.86(0.79,0.94)^{* *}$ & $1.11(0.95,1.29)$ \\
\hline Behavioral (B) & $1.03(0.95,1.13)$ & $6.89(5.99,7.93) * * *$ & $1.30(1.17,1.44)^{* * *}$ & $0.92(0.78,1.10)$ \\
\hline Hyperactivity (H) & $0.77(0.71,0.83)^{* * *}$ & $1.44(1.31,1.57)$ & $6.63(5.87,7.50)^{* * *}$ & $1.94(1.59,2.35)^{* * *}$ \\
\hline Peer $(\mathrm{P})$ & $1.08(1.01,1.16)^{*}$ & $1.12(1.03,1.23)^{* *}$ & $1.40(1.29,1.53)^{* * *}$ & $2.48(2.12,2.91) * * *$ \\
\hline Not prosocial (nP) & $1.23(1.13,1.33)^{* * *}$ & $1.46(1.33,1.60)^{* * *}$ & $1.15(1.05,1.26)^{* *}$ & $1.99(1.71,2.33)^{* * *}$ \\
\hline $\begin{array}{l}\text { Largest subscale } \\
\text { predictor }\end{array}$ & $\begin{array}{lllll}\mathbf{E} & \mathrm{nP} & \underline{\mathrm{B}} & \mathrm{P} & \mathrm{H}\end{array}$ & B $\underline{\mathrm{nP} \quad \mathrm{H}} \quad \underline{\mathrm{P}} \mathrm{E}$ & $\begin{array}{lllll}\mathrm{H} & \underline{\mathrm{B}} \quad \mathrm{nP} & \mathrm{E}\end{array}$ & $\begin{array}{lllll}\underline{\mathbf{P}} & \mathbf{n P} & \mathrm{H} & \underline{\mathrm{E}} & \mathrm{B}\end{array}$ \\
\hline Internalizing (Int) & $3.01(2.78,3.26)^{* * *}$ & $1.18(1.09,1.28)^{* * *}$ & $1.03(0.96,1.12)$ & $2.10(1.80,2.46) * * *$ \\
\hline Externalizing (Ext) & $0.77(0.72,0.83)^{* * *}$ & $4.66(4.20,5.17)^{* * *}$ & $6.71(5.97,7.54) * * *$ & $1.65(1.40,1.94)^{* * *}$ \\
\hline Not prosocial (nP) & $1.13(1.05,1.22)^{* * *}$ & $1.87(1.71,2.03)^{* * *}$ & $0.97(0.90,1.06)$ & $1.97(1.70,2.28) * * *$ \\
\hline $\begin{array}{l}\text { Largest subscale } \\
\text { predictor }\end{array}$ & Int $n P \quad$ Ext & Ext nP Int & Ext Int nP & $\underline{\text { Int }} \quad \mathbf{n P}$ Ext \\
\hline
\end{tabular}

$* \mathrm{p}<0.05, * * \mathrm{p}<0.01, * * * \mathrm{p}<0.001$. Odds ratios presented for probability of DAWBA diagnosis per one standard deviation increase in the SDQ subscale in question. Results in bold are the hypothesized association for the disorder in question. Below the odds ratios, the five subscales are presented in order of magnitude; subscales sharing an underline were not significantly different at $\mathrm{p}<0.05$. Note that the prosocial score is reverse-scored to facilitate comparisons of effect sizes. All subscales are entered in the model as predictors. $\mathrm{N}$ is the number of observations included in the model

that need to be taken into account, and that a too simplified approach may not be appropriate when dealing with patients in real-world settings. As reported by Goodman et al. [5], discriminating symptom clusters may be easier when focusing on children with more severe mental health problems (i.e., a clinical population) and this differentiation may be more difficult to establish when levels of psychopathology are low (like in epidemiological samples).
It is possible that the expression, perception, and report of symptoms in low risk samples might be unspecific and blurred, whereas in high-risk samples the specificity of symptoms for each disorder increases. Such a pattern would inevitably influence the factorial structure of symptoms. Additionally, epidemiological studies have generally missed out participants suffering from serious mental health problems or otherwise disadvantaged [56]. In our 


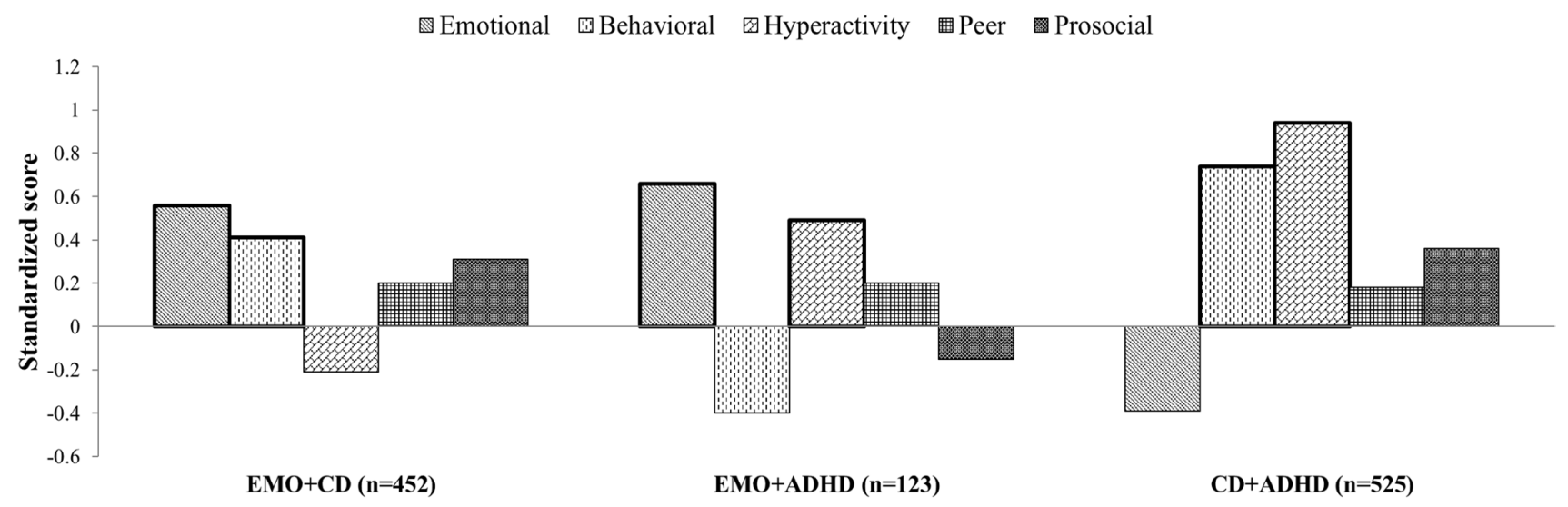

Fig. 3 Standardized SDQ subscale scores across different comorbidities. Bars with bold border are those scores hypothesized to be higher for that group. $A D H D$ attention-deficit/hyperactivity disorder, $C D$ conduct disorder, $E M O$ emotional disorders

study, mean SDQ Total Difficulties scores ranged between 16.3 and 18.3. In contrast, mean scores in epidemiological studies never reach these levels of severity, with average scores ranging around 7.5-11.0 points [e.g., 57, 58].

It could be argued that our findings, which contrast with those obtained in epidemiological studies, are due to referral biases in our samples. However, even if such biases were operating, it would not take away from the fact that a substantial proportion of severely impaired young people- those who attend clinics-show a structure of psychopathological symptoms that is different from that observed in epidemiological studies. Moreover, it would be expected that at least some of the referral biases would be different between England and Norway-yet, we demonstrate strict factorial invariance across the two samples. This becomes especially relevant when taking into account that the two countries under study have shown differences in the presentation of their psychiatric symptoms, also measured by the SDQ [e.g., 25, 59]. MI analyses also showed that, at least in clinical samples, psychiatric symptoms cluster in these five factors in boys and girls of different ages. Our results suggest that, given a certain level of severity, groups of symptoms might be already defined from early stages in the development with no distinctions across genders. This would be in line with previous research showing that psychopathology appears to be differentiated among younger children as much as it is among older children [60]. Interestingly, in a recent study across five European countries using an epidemiological sample of adolescents $(\mathrm{N}=3012)$ which also used the SDQ [57], MI across countries was only partial (11 items out of 25 were invariant), suggesting that some items should be considered carefully when using across countries. However, this study used the self-reported version of the questionnaire and participants were older than those in our sample (mean age $=14.20$ ). Authors point out that the developmental changes that occur in adolescence could be different depending on factors such as the geographical area, the culture, and the meaning of the items or the language.

A more granular approach to psychiatric symptoms, where several dimensions of symptoms are considered, also shows to be helpful when predicting psychopathology. In keeping with our hypotheses, the split between SDQ subscales allowed for distinctions between disorders, even when other disorders were also present. This finding is relevant for etiological studies. Twin studies and risk-factor studies have shown that there are substantial phenotypic correlations among pairs of psychiatric disorders that are influenced by the same genetic factors [e.g., 61, 62]. This indicates that causes of the disorders may be similar and seems to encourage a transdiagnostic approach to psychiatric disorders [4]. Additionally, this more parsimonious approach may be helpful when looking at low-risk samples [5] or when studying correlates, neural mechanisms, outcomes that are common across mental disorders [3], and factors of resilience to psychiatric disorder [33]. However, in clinical samples and when looking for models that can inform clinical predictions and treatment choices, a model considering a broader range of symptom dimensions could be more accommodating. The differences in treatment response across seemingly related behaviors/symptoms [16-19] suggest that, in order to understand the genetic or neural substrates of psychopathology, we should probably use more specific models which split patterns of symptoms. Clinicians can benefit from this approach to more sensitively screen patients referred to mental health services. An instrument as short as the SDQ seems to be helpful in making distinctions provided its multi-dimensional structure is retained. However, it is important to note that these multiple dimensions are still grouping together a number of conditions (e.g., the emotional scale of the SDQ may contain a range of anxiety and depressive disorders that may require different treatment approaches) and, hence, an in depth assessment that takes into account the specificity 
of psychopathology at the diagnostic level is still preferred if time and resources allow.

The results of this study need to be considered in light of its limitations. First, we only used parent-reported measures in our analyses. However, those are typically collected in pediatric populations, especially in younger children. Second, the structure of psychopathology may be, to some extent, influenced by the type of instrument used. Hence, we cannot rule out the possibility that different instruments could lead to different results. Studies in adult studies have used different assessment methods and, hence, a head to head comparison between pediatric and adult samples may be difficult. However, the pediatric studies with which we are comparing our results have also used the SDQ. Third, each section of the DAWBA uses skip-rules, one component of which is in some occasions the relevant SDQ subscale. For example, in the hyperactivity disorder section, parents positively reporting 'some problems with hyperactivity or poor concentration' or an SDQ hyperactivity score $\geq 6$ for their child, will continue responding items in the section; otherwise, they will be directed to the next section. Therefore, predicting DAWBA diagnoses using the SDQ subscale scores might be somewhat circular. However, in order to test to what extent circularity would have affected our results, we performed additional analyses (see Supplemental Material) using the Avon longitudinal study of parents and children (ALSPAC) sample [63], where SDQ skip rules were not employed to define DAWBA diagnoses. The results of these analyses showed that using SDQ skip rules to define diagnoses-against not using these rules-did not modify significantly the predictions. Most importantly, the specificity of predictions between SDQ subscales and relevant diagnoses was clear in both approaches.

\section{Summary}

It has been suggested that the structure of psychiatric disorders can be reduced to a few symptom dimensions. These proposals, mainly based on epidemiological samples, may not apply to clinical populations. We aimed to test the structure of psychiatric symptoms across two pediatric clinical samples from different countries and to ascertain the predictive value of each factor within them. Two clinical samples from England ( $\mathrm{N}=8434)$ and Norway $(\mathrm{N}=5866)$-assessed with the parent-reported SDQ and the DAWBA-were used to test our aims. Confirmatory factor analyses of the parentreported SDQ evaluated the relative fit of several models, including a first-order model, a second-order model with the widely-established broad symptom dimensions of internalizing-externalizing, and two bi-factor models capturing a general psychopathology factor. Measurement invariance was examined to establish whether both samples were meaningfully comparable. Predictive value of the SDQ subscales for psychiatric disorders was examined through logistic regressions. A first-order five-factor solution better fit the data in both samples. Measurement invariance held for all comparisons by gender, age, and country. In all cases, the expected SDQ subscale(s) related best to the corresponding DAWBA diagnosis (e.g., emotional to emotional disorder; conduct problems to conduct disorder; hyperactivity to ADHD), even in cases of comorbidity. Our findings suggest that, in clinical samples, a more granular approach to psychiatric symptoms where several dimensions are considered seems to fit the data better than a model based alone on splitting between internalizing/externalizing dimensions. This approach is also helpful when predicting psychopathology. Future research needs to examine whether this factor structure holds across other clinical samples and other psychometric measures using a variety of informants (including teachers and the patients themselves).

Acknowledgements We are extremely grateful to Dr. Anna Goodman for her support in provision and preparation of data analysis scripts for confirmatory factor analyses.

Funding Dr. Fernández de la Cruz and Mr. Vidal-Ribas were fully supported, and Dr. Simonoff and Dr. Stringaris were partly supported, by the National Institute for Health Research (NIHR) Biomedical Research Centre for Mental Health at South London and Maudsley National Health Service (NHS) Foundation Trust and the Institute of Psychiatry, King's College London. Mr. Vidal-Ribas was also supported by the Alicia Koplowitz Foundation. Both Mr. Vidal-Ribas and Dr. Stringaris were supported by the NIMH Intramural Research Program. Dr. Stringaris, before assuming his duties at NIMH in 2016, had received funding from the Wellcome Trust and the UK National Institute of Health Research, funds from University College London for a joint project with Johnson \& Johnson and royalties from Cambridge University Press and Oxford University Press. The rest of the authors report no financial relationships with commercial interests. BRC Clinical Records Interactive Search (CRIS) is supported by the National Institute for Health Research (NIHR) Biomedical Research Centre for Mental Health BRC Nucleus at the South London and Maudsley NHS Foundation Trust and Institute of Psychiatry, King's College London jointly funded by the Guy's and St Thomas' Trustees and the South London and Maudsley Trustees.

\section{Compliance with Ethical Standards}

Conflict of interest Dr. Fernández de la Cruz receives royalties for contributing articles to UpToDate, Wolters Kluwer Health. Prof. Goodman is the owner of Youthinmind Ltd, which provides no-cost and low-cost websites related to the Strengths and Difficulties Questionnaire and the Development and Well-Being Assessment. The authors have no other competing interests to declare.

Open Access This article is distributed under the terms of the Creative Commons Attribution 4.0 International License (http://creativecommons.org/licenses/by/4.0/), which permits unrestricted use, distribution, and reproduction in any medium, provided you give appropriate credit to the original author(s) and the source, provide a link to the Creative Commons license, and indicate if changes were made. 


\section{References}

1. Kendler KS, Prescott CA, Myers J, Neale MC (2003) The structure of genetic and environmental risk factors for common psychiatric and substance use disorders in men and women. Arch Gen Psychiatry 60:929-937

2. Wright AG, Krueger RF, Hobbs MJ, Markon KE, Eaton NR, Slade T (2013) The structure of psychopathology: toward an expanded quantitative empirical model. J Abnormal Psychol 122:281-294

3. Lahey BB, Applegate B, Hakes JK, Zald DH, Hariri AR, Rathouz PJ (2012) Is there a general factor of prevalent psychopathology during adulthood? J Abnormal Psychol 121:971-977

4. Caspi A, Houts RM, Belsky DW, Goldman-Mellor SJ, Harrington $\mathrm{H}$, Israel S et al (2014) The p factor: one general psychopathology factor in the structure of psychiatric disorders? Clin Psychol Sci $2: 119-137$

5. Goodman A, Lamping DL, Ploubidis GB (2010) When to use broader internalising and externalising subscales instead of the hypothesised five subscales on the strengths and difficulties questionnaire (SDQ): data from British parents, teachers and children. J Abnorm Child Psychol 38:1179-1191

6. Goodman R (1997) The strengths and difficulties questionnaire: a research note. J Child Psychol Psychiatry 38:581-586

7. Goodman R (2001) Psychometric properties of the strengths and difficulties questionnaire. J Am Acad Child Adolesc Psychiatry 40:1337-1345

8. Achenbach TM, Edelbrock CS (1978) The classification of child psychopathology: a review and analysis of empirical efforts. Psychol Bull 85:1275-1301

9. Cross-Disorder Group of the Psychiatric Genomics Consortium (2013) Identification of risk loci with shared effects on five major psychiatric disorders: a genome-wide analysis. Lancet 381:1371-1379

10. Rutter M (2011) Research review: child psychiatric diagnosis and classification: concepts, findings, challenges and potential. J Child Psychol Psychiatry 52:647-660

11. Kotov R, Ruggero CJ, Krueger RF, Watson D, Yuan Q, Zimmerman M (2011) New dimensions in the quantitative classification of mental illness. Arch Gen Psychiatry 68:1003-1011

12. Becker A, Woerner W, Hasselhorn M, Banaschewski T, Rothenberger A (2004) Validation of the parent and teacher SDQ in a clinical sample. Eur Child Adolesc Psychiatry 13(Suppl 2):II11-I16

13. Becker A, Hagenberg N, Roessner V, Woerner W, Rothenberger A (2004) Evaluation of the self-reported SDQ in a clinical setting: do self-reports tell us more than ratings by adult informants? Eur Child Adolesc Psychiatry 13(Suppl 2):II17-I24

14. Fabrigar LR, Wegener DT, MacCallum RC, Strahan EJ (1999) Evaluating the use of exploratory factor analysis in psychological research. Psychol Methods 4:272

15. Pine D (2011) Commentary: diagnosis and classification: there must be something left about which to argue-reflections on Rutter (2011). J Child Psychol Psychiatry 52:663-664 (discussion 673-665)

16. Daley D, van der Oord S, Ferrin M, Danckaerts M, Doepfner $\mathrm{M}$, Cortese S et al (2014) Behavioral interventions in attentiondeficit/hyperactivity disorder: a meta-analysis of randomized controlled trials across multiple outcome domains. J Am Acad Child Adolesc Psychiatry 53:835-847

17. Pilling S, Gould N, Whittington C, Taylor C, Scott S, Guideline Development Group (2013) Recognition, intervention, and management of antisocial behaviour and conduct disorders in children and young people: summary of NICE-SCIE guidance. BMJ 346:f 1298
18. Fernández de la Cruz L, Simonoff E, McGough JJ, Halperin JM, Arnold LE, Stringaris A (2015) Treatment of children with attention-deficit/hyperactivity disorder (ADHD) and irritability: results from the multimodal treatment study of children with ADHD (MTA). J Am Acad Child Adolesc Psychiatry 54:62-70

19. Galanter CA, Carlson GA, Jensen PS, Greenhill LL, Davies M, $\mathrm{Li} \mathrm{W}$ et al (2003) Response to methylphenidate in children with attention deficit hyperactivity disorder and manic symptoms in the multimodal treatment study of children with attention deficit hyperactivity disorder titration trial. J Child Adolesc Psychopharmacol 13:123-136

20. Goodman R, Renfrew D, Mullick M (2000) Predicting type of psychiatric disorder from strengths and difficulties questionnaire (SDQ) scores in child mental health clinics in London and Dhaka. Eur Child Adolesc Psychiatry 9:129-134

21. Mathai J, Anderson P, Bourne A (2004) Comparing psychiatric diagnoses generated by the strengths and difficulties questionnaire with diagnoses made by clinicians. Aust $\mathrm{N} Z \mathrm{~J}$ Psychiatry 38:639-643

22. Brøndbo PH, Mathiassen B, Martinussen M, Heiervang E, Eriksen M, Moe TF et al (2011) The strengths and difficulties questionnaire as a screening instrument for norwegian child and adolescent mental health services, application of UK scoring algorithms. Child Adolesc Psychiatry Ment Health 5:32

23. Achenbach TM, Becker A, Dopfner M, Heiervang E, Roessner V, Steinhausen HC et al (2008) Multicultural assessment of child and adolescent psychopathology with ASEBA and SDQ instruments: research findings, applications, and future directions. J Child Psychol Psychiatry 49:251-275

24. Stone LL, Otten R, Engels RC, Vermulst AA, Janssens JM (2010) Psychometric properties of the parent and teacher versions of the strengths and difficulties questionnaire for 4- to 12-year-olds: a review. Clin Child Fam Psychol Rev 13:254-274

25. Heiervang E, Goodman A, Goodman R (2008) The Nordic advantage in child mental health: separating health differences from reporting style in a cross-cultural comparison of psychopathology. J Child Psychol Psychiatry 49:678-685

26. Fernandes AC, Cloete D, Broadbent MT, Hayes RD, Chang CK, Jackson RG et al (2013) Development and evaluation of a deidentification procedure for a case register sourced from mental health electronic records. BMC Med Inform Decis Mak 13:71

27. Stewart R, Soremekun M, Perera G, Broadbent M, Callard F, Denis M et al (2009) The South London and Maudsley NHS Foundation Trust Biomedical Research Centre (SLAM BRC) case register: development and descriptive data. BMC Psychiatry 9:51

28. Chang CK, Hayes RD, Perera G, Broadbent MTM, Fernandes AC, Lee WE et al (2011) Life expectancy at birth for people with serious mental illness and other major disorders from a secondary mental health care case register in London. PloS ONE 6:e19590

29. Fernández de la Cruz L, Llorens M, Jassi A, Krebs G, VidalRibas P, Radua J et al (2015) Ethnic inequalities in the use of secondary and tertiary mental health services amongst patients with obsessive-compulsive disorder. Br J Psychiatry 207:530-535

30. Wu CY, Chang CK, Hayes RD, Broadbent M, Hotopf M, Stewart R (2012) Clinical risk assessment rating and all-cause mortality in secondary mental healthcare: the South London and Maudsley NHS Foundation Trust Biomedical Research Centre (SLAM BRC) Case Register. Psychol Med 42:1581-1590

31. Stringaris A, Goodman R (2013) The value of measuring impact alongside symptoms in children and adolescents: a longitudinal assessment in a community sample. J Abnorm Child Psychol 41:1109-1120

32. Obel C, Heiervang E, Rodriguez A, Heyerdahl S, Smedje H, Sourander A et al (2004) The strengths and difficulties questionnaire in the Nordic countries. Eur Child Adolesc Psychiatry 13(Suppl 2):II32-I39 
33. Vidal-Ribas P, Goodman R, Stringaris A (2015) Positive attributes in children and reduced risk of future psychopathology. $\mathrm{Br} \mathrm{J}$ Psychiatry 206:17-25

34. Goodman R, Ford T, Richards H, Gatward R, Meltzer H (2000) The development and well-being assessment: description and initial validation of an integrated assessment of child and adolescent psychopathology. J Child Psychol Psychiatry 41:645-655

35. American Psychiatric Association (1994) The diagnostic and statistical manual of mental disorders: (DSM-IV), 4th edn. Washington, DC, American Psychiatric Association

36. Meltzer H, Gatward R, Goodman R, Ford T (2000) Mental health of children and adolescents in Great Britain. TSO, London

37. Goodman A, Heiervang E, Collishaw S, Goodman R (2011) The 'DAWBA bands' as an ordered-categorical measure of child mental health: description and validation in British and Norwegian samples. Soc Psychiatry Psychiatr Epidemiol 46:521-532

38. Stringaris A, Vidal-Ribas Belil P, Artiges E, Lemaitre H, GollierBriant F, Wolke S et al (2015) The brain's response to reward anticipation and depression in adolescence: dimensionality, specificity, and longitudinal predictions in a community-based sample. Am J Psychiatry 172:1215-1223

39. Ford T, Goodman R, Meltzer H (2003) The British child and adolescent mental health survey 1999: the prevalence of DSM-IV disorders. J Am Acad Child Adolesc Psychiatry 42:1203-1211

40. Muthén B (1983) Latent variable structural equation modeling with categorical data. J Econom 22:43-65

41. Muthén B (1984) A general structural equation model with dichotomous, ordered categorical, and continuous latent variable indicators. Psychometrika 49:115-132

42. Hu L, Bentler PM (1999) Cutoff criteria for fit indexes in covariance structure analysis: conventional criteria versus new alternatives. Struct Equ Modeling 6:1-55

43. Brown T (2006) Confirmatory factor analysis for applied research. The Guilford Press, New York

44. Burnham KP, Anderson DR (2004) Multimodel inference: understanding AIC and BIC in model selection. Sociol Method Res 33:261-304

45. Levy R, Hancock GR (2007) A framework of statistical tests for comparing mean and covariance structure models. Multivar Behav Res 42:33-66

46. Bollen KA (1989) A new incremental fit index for general structural equation models. Sociol Method Res 17:303-316

47. Cheung GW, Rensvold RB (2002) Evaluating goodness-of-fit indexes for testing measurement invariance. Struct Equ Modeling 9:233-255

48. Jöreskog KG (1971) Simultaneous factor analysis in several populations. Psychometrika 36:409-426
49. Vandenberg RJ, Lance CE (2000) A review and synthesis of the measurement invariance literature: suggestions, practices, and recommendations for organizational research. Organ Res Methods $3: 4-70$

50. Little TD (1997) Mean and covariance structures (MACS) analyses of cross-cultural data: practical and theoretical issues. Multivar Behav Res 32:53-76

51. Meredith W, Teresi JA (2006) An essay on measurement and factorial invariance. Med Care 44:S69-S77

52. Cochran WG (1952) The $\chi 2$ test of goodness of fit. Ann Math Stat 23:315-345

53. Brannick MT (1995) Critical comments on applying covariance structure modeling. J Organ Behav 16:201-213

54. Schafer JL, Graham JW (2002) Missing data: our view of the state of the art. Psychol Methods 7:147-177

55. Byrne BM (2013) Structural equation modeling with Mplus: basic concepts, applications, and programming. Routledge, New York

56. Knudsen AK, Hotopf M, Skogen JC, Overland S, Mykletun A (2010) The health status of nonparticipants in a populationbased health study: the Hordaland Health Study. Am J Epidemiol 172:1306-1314

57. Ortuño-Sierra J, Fonseca-Pedrero E, Aritio-Solana R, Velasco AM, de Luis EC, Schumann G et al (2015) New evidence of factor structure and measurement invariance of the SDQ across five European nations. Eur Child Adolesc Psychiatry 24:1-12

58. Peacock PJ, Lewis G, Northstone K, Wiles NJ (2011) Childhood diet and behavioural problems: results from the ALSPAC cohort. Eur J Clin Nutr 65:720-726

59. Goodman A, Heiervang E, Fleitlich-Bilyk B, Alyahri A, Patel V, Mullick MS et al (2012) Cross-national differences in questionnaires do not necessarily reflect comparable differences in disorder prevalence. Soc Psychiatry Psychiatr Epidemiol 47:1321-1331

60. Sterba S, Egger HL, Angold A (2007) Diagnostic specificity and nonspecificity in the dimensions of preschool psychopathology. J Child Psychol Psychiatry 48:1005-1013

61. Kendler KS (1996) Major depression and generalised anxiety disorder: same genes, (partly)different environments-revisited. Br J Psychiatry Suppl 30:68-75

62. Lichtenstein P, Yip BH, Bjork C, Pawitan Y, Cannon TD, Sullivan $\mathrm{PF}$ et al (2009) Common genetic determinants of schizophrenia and bipolar disorder in Swedish families: a population-based study. Lancet 373:234-239

63. ALSPAC Study Team (2001) ALSPAC-the Avon longitudinal study of parents and children. Paediatr Perinat Epidemiol 15:74-87 